DOI: $10.20472 /$ IAC.2019.048.019

\author{
LI-FEI HUANG \\ Ming Chuan University, Taiwan
}

\title{
USING APP INVENTOR TO PROVIDE THE ONE-WAY ANOVA TABLE WITH BLOCKS
}

\begin{abstract}
:
The one-way ANOVA table is used to test whether the factor has some influence on the experimental result or not. The factor has at least two levels, and some uncontrollable block usually appears in the experiment. An engineering calculator can help computing different sum of squares, but the ANOVA table must be written or typed in somewhere else. By the way, a smart phone is more convenient than a calculator or a tablet. Rather than the traditional spreadsheet, the free MIT App Inventor is applied to create the one-way ANOVA table with blocks in the mobile computing environment in this paper.
\end{abstract}

\section{Keywords:}

App Inventor, one-way ANOVA, block, financial technique.

JEL Classification: Y10 